Proceedings of the 8th Workshop on Quantum Chaos and Localisation Phenomena, May 19-21, 2017, Warsaw, Poland

\title{
Multifractality in Fock Space of the Ground State of the Bose-Hubbard Hamiltonian
}

\author{
J. Lindinger And A. RodRÍGUEZ* \\ Physikalisches Institut, Albert-Ludwigs-Universität Freiburg, Hermann-Herder-Str. 3, D-79104, Freiburg, Germany

\begin{abstract}
We present a first study of the multifractal properties of the ground state of the Bose-Hubbard Hamiltonian in Fock space. Numerical simulations of system sizes up to $L=10$ at unit filling suggest that multifractality is present for all values of the bosonic interaction strength. Moreover, the analysis of the generalised fractal dimensions for different densities exposes qualitatively the superfluid to Mott insulator phase transition.
\end{abstract}

DOI: 10.12693/APhysPolA.132.1683

PACS/topics: multifractality, localisation in Fock space, quantum phase transitions

\section{Introduction}

The wave function of a quantum system in Hilbert space can exhibit an unexpected degree of complexity, even for apparently simple Hamiltonians. One example of such complexity is multifractality, which endows the quantum states with unusual statistical properties [1,2]: due to the fact that a multifractal is a composition of multiple fractals with different fractal dimensions, the moments of a multifractal distribution obey an anomalous scaling law. Multifractal wave functions were first found at the critical point of the disorder-induced localisation-delocalisation transition (the Anderson transition) in single-particle Hamiltonians [3, 4]. The multifractal analysis of the properties of these critical quantum states has turned out to be an extremely useful tool to discover the physics behind this quantum phase transition (see Ref. [5] and references therein).

The significance of multifractality in quantum systems, however, goes well beyond non-interacting systems. It was recently shown that multifractality at the Anderson transition remains in the presence of Coulomb interactions $[6,7]$. Multifractality also seems to play a prominent role in many-body systems subject to strong disorder $[8,9]$, in which an insulating ("many-body localised") phase can emerge, corresponding to a many-particle wave function which is localised in Fock space [10-12]. Perhaps most striking is the observation that the ground state of certain many-body spin systems exhibits multifractality in Hilbert space in the absence of any disorder $[13,14]$. Moreover, different quantum phases in these spin systems can be exposed by studying corrections to multifractality [15].

Wave function multifractality in Hilbert space seems to be a generic feature of many-body systems. Even in the absence of disorder the energetic landscape in Hilbert space is inhomogeneous, which makes ergodicity unlikely.

\footnotetext{
* corresponding author; e-mail:

Alberto.Rodriguez.Gonzalez@physik.uni-freiburg.de
}

Additionally, the connectivity among the "nodes" (i.e. basis states) in Hilbert space grows with system size, which renders localisation also improbable. Hence, multifractal states, which are delocalised but occupy a vanishing fraction of the total space in the thermodynamic limit (i.e. non-ergodic), may be predominant. This in turn raises the interesting question of whether the features of different phases in many-body systems can be seen through the multifractal properties in Hilbert space.

Here, we present a first study of the features of the ground state wave function of a system of many interacting bosons in Fock space. We provide evidence for the existence of multifractality for all values of the interaction strength, and show that it is distinctly different depending on whether the system is in a superfluid or a Mott insulating phase. We review briefly the multifractal formalism in Sect. 2, and present our results and conclusions in Sects. 3 and 4.

\section{Multifractal formalism}

Let us consider a wave function, $|\Psi\rangle$, expanded in a given eigenbasis, $\{|j\rangle\}$, of a finite Hilbert space of dimen$\operatorname{sion} \mathcal{N}$,

$$
|\Psi\rangle=\sum_{j=1}^{\mathcal{N}} \psi_{j}|j\rangle .
$$

The distribution of probabilities $\left|\psi_{j}\right|^{2}$ is said to be multifractal if its $q$-moments, $R_{q} \equiv \sum_{j}\left|\psi_{j}\right|^{2 q}$ for $q \in \mathbb{R}$, obey the following scaling law

$$
R_{q} \sim \mathcal{N}^{-\tau_{q}}, \text { for } \mathcal{N} \rightarrow \infty,
$$

where the exponents $\tau_{q}$ must depend non-linearly on $q$, and are usually written in the form

$$
\tau_{q}=D_{q}(q-1)
$$
and $D_{q}$ are called the generalised fractal dimensions.

\footnotetext{
$\ddagger$ In non-interacting models, where the dimension of Hilbert space relates to the linear size $L$ of the system as a power-law, e.g. as in the Anderson model defined on a $d$-dimensional lattice $\left(\mathcal{N}=L^{d}\right)$, the scaling law given in Eq. (2) is usually written in terms of $L$, rather than the dimensionality of Hilbert space.
} 
The exponents $\tau_{q}$ are related to the so-called multifractal spectrum $f(\alpha)$ via a Legendre transformation [16],

$$
\begin{aligned}
& f\left(\alpha_{q}\right)=q \alpha_{q}-\tau_{q}, \\
& \alpha_{q} \equiv \frac{\mathrm{d} \tau_{q}}{\mathrm{~d} q} .
\end{aligned}
$$

The multifractal spectrum is a continuous and differentiable function of the singularity strength $\alpha$, and it has the following meaning: $f(\alpha)$ gives the fractal dimension of the set of points in the wave function with the same intensity, characterised by the singularity strength $\alpha=-\ln \left|\psi_{j}\right|^{2} / \ln \mathcal{N}$. A multifractal wave function is thus the result of a composition of different fractal layers, each of which correlates with a given possible value of the intensity $\left|\psi_{j}\right|^{2}$. Observationally, a multifractal eigenstate exhibits an intricate fluctuating pattern which extends throughout Hilbert space, as shown in Fig. 1.

In quantum systems, multifractality appears most prominently at the critical point of disorder-induced localisation-delocalisation transitions [5]. In this context, scaling law (2) can also be used to discern the localised and extended characters of a wave function in Hilbert space: for an ergodic state (i.e. a state with homogeneous intensities $\left|\psi_{j}\right|^{2} \sim \mathcal{N}^{-1}$ as $\mathcal{N} \rightarrow \infty$ ) the generalised multifractal dimensions have the trivial values $D_{q}=1$, for all $q$. On the other hand, localisation implies that only a finite subset of basis states contributes to the wave function, and hence the moments $R_{q}$ become independent of $\mathcal{N}$ as $\mathcal{N} \rightarrow \infty$, leading to $D_{q}=0$ for $q>0$. Multifractality is then exposed by non-trivial values of the generalised multifractal dimensions $0<D_{q}<1$. (Note that $D_{0}$ is 1 for any normalised distribution.)

For the numerical characterisation of multifractality, it is convenient to define the $\mathcal{N}$-dependent dimensions

$$
\widetilde{D}_{q} \equiv \frac{1}{1-q} \frac{\ln R_{q}}{\ln \mathcal{N}},
$$

which converge to the generalised fractal dimensions with increase of $\mathcal{N}$,

$$
D_{q}=\lim _{\mathcal{N} \rightarrow \infty} \widetilde{D}_{q} .
$$

The use of $\widetilde{D}_{q}$ and of other related $\mathcal{N}$-dependent multifractal exponents allows to generalise scaling laws of type (2) in order to describe the persistence of wave function multifractality in the vicinity of the Anderson transition. Such a generalised multifractal analysis has been used to perform high-precision studies of the critical properties of the transition in the non-interacting case [17-22]. This formalism is also potentially applicable in the presence of interactions, and work along this line is currently being pursued [23, 24].

\section{The ground state of the Bose-Hubbard Hamiltonian}

The Bose-Hubbard Hamiltonian (BHH), which can be faithfully implemented experimentally, describes interacting bosons restricted to the first energy band of an optical lattice [26-29]:
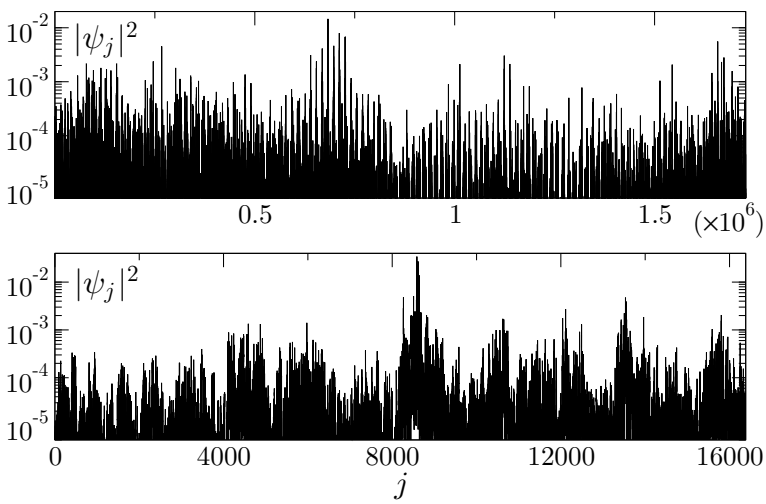

Fig. 1. Plot of multifractal wave functions versus the index $j$ of the basis elements in Hilbert space, for two non-interacting models: (top) At the critical point of the 3D Anderson transition for a cubic system of linear size $L=120$. (bottom) Critical eigenstate of an ultrametric random matrix [25] of size $\mathcal{N}=2^{14}$.

$$
H=-J \sum_{\langle k, j\rangle} b_{k}^{\dagger} b_{j}+\frac{U}{2} \sum_{k=1}^{L} n_{k}\left(n_{k}-1\right),
$$

in terms of bosonic creation/annihilation operators $b_{k}^{\dagger}$, $b_{k}, n_{k}=b_{k}^{\dagger} b_{k}$, in a one-dimensional lattice of $L$ sites. Hopping (characterised by the hopping strength $J$ ) is restricted to nearest neighbours, and we consider a repulsive on-site interaction, $U>0$.

For fixed density $\nu=N / L$, Hilbert space grows exponentially with the system size $L$ (i.e. with the number of particles $N)$,

$$
\mathcal{N}=\left(\begin{array}{c}
N+L-1 \\
N
\end{array}\right)_{N, L \gg 1}^{\sim} \frac{1}{\sqrt{L}}\left[\frac{(\nu+1)^{\nu+1}}{\nu^{\nu}}\right]^{L} .
$$

A basis of Hilbert space is given by Fock states of the on-site density operators, $|\boldsymbol{n}\rangle \equiv\left|n_{1}, n_{2}, \ldots, n_{L}\right\rangle$. Hence the ground state of the system can be expanded as

$$
|\Psi\rangle=\sum_{\boldsymbol{n}} \psi_{\boldsymbol{n}}|\boldsymbol{n}\rangle .
$$

In the non-interacting case $(U=0)$ all bosons occupy the lowest energy single-particle Bloch state, and in the thermodynamic limit $(L \rightarrow \infty, N \rightarrow \infty)$ the energy cost to induce an excitation in the system goes to zero. In contradistinction, when $U \rightarrow \infty$ for integer bosonic density $\nu$ the ground state is given by one Fock state, with a fixed number $\nu$ of bosons per site. In this case the simplest excitation consists in moving one boson to a neighbouring site, which entails as a minimum energy cost the interaction energy $U$. For integer filling factor, the ground state of the system undergoes a phase transition in the thermodynamic limit at a finite value of the interaction strength, $(U / J)_{c}$, between two phases, a superfluid (SF) phase and a Mott insulator (MI) phase [30-32], which keep some of the properties of the two limiting cases discussed ${ }^{\dagger}$. In

\footnotetext{
$\dagger^{\dagger}$ The transition can also be driven by a change in the bosonic
} 
the one-dimensional system, this interaction-driven transition at fixed density is of the Kosterlitz-Thouless type (it lies in the universality class of the two-dimensional $X Y$ spin model). The transition can be characterised using different figures of merit [26]: the SF phase is gapless (vanishing excitation energy), it has non-vanishing superfluid fraction and an infinite correlation length. On the other hand, the MI phase is gapped, the superfluid fraction vanishes and the correlation length is finite. Additionally, the on-site density fluctuations are strongly suppressed in Mott insulator [33, 34].

As discussed above, the ground state in the limit $U \rightarrow$ 0 involves a superposition of all Fock states, while for $U \rightarrow \infty$ we have $|\Psi\rangle=|\nu, \nu, \ldots, \nu\rangle$. Hence, the SF to $\mathrm{MI}$ transition resembles a delocalisation-localisation transition in Fock space. This perspective suggests that the analysis of multifractality in Fock space could provide some additional insight into this transition. Indeed, as shown in Fig. 2, the visualisation of the ground state in Fock basis resembles that of the critical states of Fig. 1. This indicates that the ground state could be multifractal in Fock basis for certain values of the interaction.

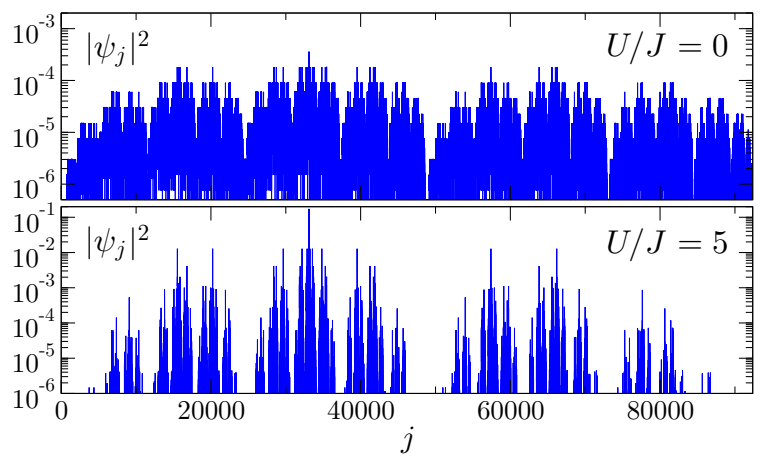

Fig. 2. Plot of the ground state wave function of the 1D BHH versus the index $j$ of the basis elements $|\boldsymbol{n}\rangle$ in Fock space, for $L=N=10(\mathcal{N}=92378)$ and two different values of the interaction strength $U$. Note that the indexing function $j=j(|\boldsymbol{n}\rangle)$ of the basis elements, while affecting the visualization of the wavefunction, is irrelevant for the multifractal analysis described in the text.

In the non-interacting case, it is possible to find an analytical expression for the ground state in Fock basis

$$
\left|\Psi_{U=0}\right\rangle=\sum_{\boldsymbol{n}} \sqrt{\frac{N !}{L^{N} n_{1} ! n_{2} ! \ldots n_{L} !}}|\boldsymbol{n}\rangle,
$$

for a fixed number of bosons, i.e. it must be $n_{1}+\ldots+$ $n_{L}=N$, and assuming periodic boundary conditions. From the knowledge of the amplitudes $\psi_{\boldsymbol{n}}$, the analytical calculation of the moments $R_{q}=\sum_{n}\left|\psi_{\boldsymbol{n}}\right|^{2 q}$ and of $D_{q}$ is possible. The generalised fractal dimensions for $U=0$

density at fixed $U / J$; here we will only consider the interactiondriven case. These two phase transitions do not belong to the same universality class. can be obtained as

$$
D_{q}=\frac{\ln f_{q}\left(z_{c}\right)-\nu \ln z_{c}-q \nu(1-\ln \nu)}{(1-q)[(\nu+1) \ln (\nu+1)-\nu \ln \nu]},
$$

where $f_{q}(z)=\sum_{k=0}^{\infty} z^{k} /(k !)^{q}$, and $z_{c}$ is the solution of $f_{q}^{\prime}\left(z_{c}\right) / f_{q}\left(z_{c}\right)=\nu / z_{c}$. This result was first obtained by Bogomolny [35], and proves that even for vanishing interaction the ground state in Fock basis exhibits non-trivial multifractality (see black line in the inset of Fig. 3).

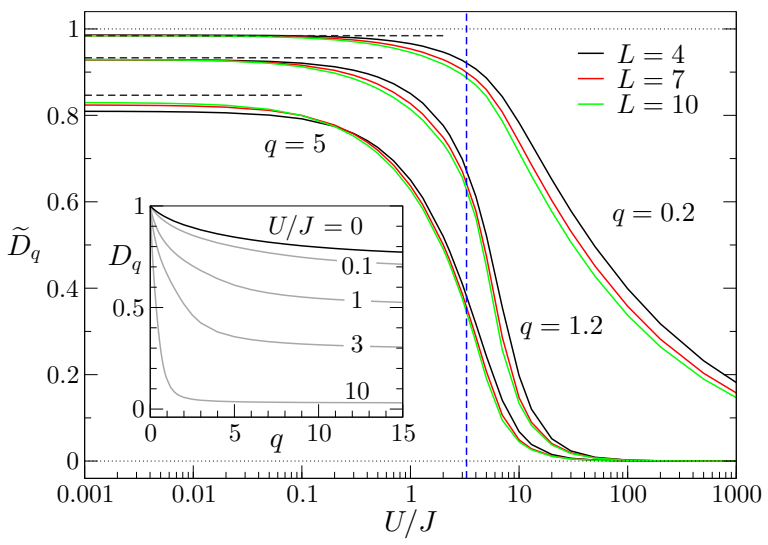

Fig. 3. System-size dependent fractal dimensions $\widetilde{D}_{q}$ versus interaction strength $U / J$ for $q=0.2,1.2,5$, for the ground state of the BHH with $\nu=1$. The horizontal dashed lines mark the value of $D_{q}$ in the limit $U \rightarrow 0$ given by Eq. (12). The vertical dashed line highlights the position of the SF to MI transition in the thermodynamic limit: $(J / U)_{c}=0.305 \pm 0.001$ [36]. The inset shows the extrapolated $(\mathcal{N} \rightarrow \infty)$ generalised fractal dimensions $D_{q}$ versus $q$ for several values of $U / J$.

For non-vanishing interaction, an exact analytical expression for the ground state cannot be obtained, and the multifractal analysis must be performed numerically. For system sizes $L \lesssim 12$, the BHH for $\nu=1$ can be treated by exact diagonalisation. In Fig. 3, we show the evolution of the $\mathcal{N}$-dependent dimensions $\widetilde{D}_{q}$ (Eq. (6)) as functions of the interaction strength, for system sizes $L=4,7,10$. The behaviour of $\widetilde{D}_{q}$ suggests that the ground state is multifractal for any finite value of $U$. This is confirmed by the generalised fractal dimensions $D_{q}$, obtained from the extrapolation $\mathcal{N} \rightarrow \infty$, shown in the inset of Fig. 3 . The $\mathcal{N}$-dependent fractal dimensions decrease monotonously with the interaction strength and they vanish as $U \rightarrow \infty$ for any $q$ value, in agreement with the localised nature of the ground state in this limit. Most interestingly, as $q$ grows, the region where the decay of the fractal dimensions is more pronounced correlates with the location of the SF to MI transition. In order to assess unambiguously whether the dimensions $D_{q}$ bear a quantifiable fingerprint of the transition, it is necessary to analyse larger system sizes, which may be accessible by quantum Monte Carlo [15].

Nonetheless, from the analysis of the system-size dependent fractal dimensions $\widetilde{D}_{q}$, even for small systems, one can clearly see a qualitative signal of the SF to MI transition. As demonstrated in Fig. 4, the fractal dimen- 
sion $\widetilde{D}_{2}$ registers a sharp decay towards zero for integer values of the bosonic density when the interaction is within the MI region. For non-integer $\nu$, however, $\widetilde{D}_{2}$ approaches asymptotically a non-zero value for increasing $U$. From what we can observe, non-trivial multifractality of the ground state is more prominent when the system is in the SF phase, as it happens for any value of the interaction whenever the density is non-integer. Note that a non-vanishing $D_{2}=\tau_{2}<1$ implies that the participation ratio, $1 / R_{2}$, of the wave function in Fock space (i.e. the region where the state is noticeable) diverges as $\mathcal{N} \rightarrow \infty$. The wave function is therefore delocalised, although it occupies a vanishing fraction of Fock space.

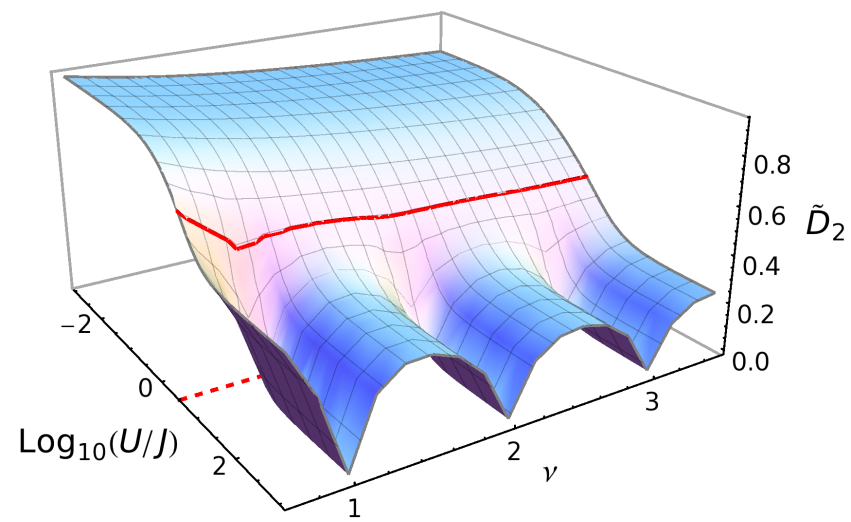

Fig. 4. Dimension $\widetilde{D}_{2}$ as a function of the interaction strength, $U / J$, and the bosonic density, $\nu$, for the ground state of the BHH with $L=6$ sites. Red lines mark the position of the the SF to MI transition in the thermodynamic limit.

\section{Conclusions}

We have presented a multifractal analysis in Fock space of the ground state of the Bose-Hubbard Hamiltonian. It can be proven analytically that non-trivial multifractality is already present in the non-interacting case. Numerical simulations of small systems using exact diagonalisation suggest that multifractality also exists for nonvanishing interaction. The degree of multifractality decreases monotonously with the interaction strength. For integer bosonic densities all fractal dimensions decay to zero as $U \rightarrow \infty$, in agreement with the emergence of Mott insulator limiting state, whereas for non-integer density, $D_{q}$ is non-vanishing for increasing interaction (cf. Ref. [35]). Our results show that the behaviour of the generalised fractal dimensions exposes qualitatively the superfluid to Mott insulator phase transition. Whether the analysis of multifractality in Fock space can also provide a quantitative characterisation of the transition remains to be investigated.

\section{Acknowledgments}

We thank Andreas Buchleitner for a careful reading of the manuscript. The authors acknowledge support by the state of Baden-Württemberg through bwHPC.

\section{References}

[1] G. Paladin, A. Vulpiani, Phys. Rep. 156, 147 (1987).

[2] B.B. Mandelbrot, J. Stat. Phys. 110, 739 (2003).

[3] H. Aoki, J. Phys. C 16, L205 (1983).

[4] H. Aoki, Phys. Rev. B 33, 7310 (1986).

[5] F. Evers, A.D. Mirlin, Rev. Mod. Phys. 80, 1355 (2008).

[6] I.S. Burmistrov, I.V. Gornyi, A.D. Mirlin, Phys. Rev. Lett. 111, 066601 (2013).

[7] I.S. Burmistrov, I.V. Gornyi, A.D. Mirlin, Phys. Rev. B 91, 085427 (2015).

[8] A. De Luca, A. Scardicchio, Europhys. Lett. 101, 37003 (2013).

[9] D.J. Luitz, N. Laflorencie, F. Alet, Phys. Rev. B 91 081103 (2015).

[10] D.M. Basko, I.L. Aleiner, B.L. Altshuler, Ann. Phys. (New York) 321, 1126 (2006).

[11] D.M. Basko, I.L. Aleiner, B.L. Altshuler, Problems of Condensed Matter Physics: On the Problem of ManyBody Localisation, Oxford University Press, Oxford 2007, p. 50.

[12] I.V. Gornyi, A.D. Mirlin, D.G. Polyakov, Phys. Rev. Lett. 95, 206603 (2005).

[13] Y.Y. Atas, E. Bogomolny, Phys. Rev. E 86, 021104 (2012).

[14] Y.Y. Atas, E. Bogomolny, Philos. Trans. R. Soc. A Math. Phys. Eng. Sci. 372, 20120520 (2013).

[15] D.J. Luitz, F. Alet, N. Laflorencie, Phys. Rev. Lett. 112, 057203 (2014).

[16] M. Janssen, Int. J. Mod. Phys. B 08, 943 (1994).

[17] A. Rodriguez, L.J. Vasquez, K. Slevin, R.A. Römer, Phys. Rev. Lett. 105, 046403 (2010).

[18] A. Rodriguez, L.J. Vasquez, K. Slevin, R.A. Römer, Phys. Rev. B 84, 134209 (2011).

[19] B.C. Huang, C.H. Chang, Phys. Rev. E 88, 042116 (2013).

[20] L. Ujfalusi, I. Varga, Phys. Rev. B 91, 184206 (2015).

[21] J. Lindinger, B.Sc. thesis, Albert-LudwigsUniversität Freiburg, 2015.

[22] J. Lindinger, A. Rodríguez, Phys. Rev. B 96, 134202 (2017).

[23] Y. Harashima, K. Slevin, Phys. Rev. B 89, 205108 (2014).

[24] E. Carnio, R.A. Römer, arXiv:1701.01742, 2017.

[25] Y.V. Fyodorov, A. Ossipov, A. Rodriguez, J. Stat. Mech. Theory Exp. 2009, L12001 (2009).

[26] M. Lewenstein, A. Sanpera, V. Ahufinger, B. Damski, A. Sen, U. Sen, Adv. Phys. 56, 243 (2007).

[27] H.T.C. Stoof, D.B.M. Dickerscheid, K. Gubbels, Ultracold Quantum Fields, Theoretical and Mathematical Physics, Springer, Dordrecht 2008.

[28] I. Bloch, J. Dalibard, W. Zwerger, Rev. Mod. Phys. 80, 885 (2008).

[29] M.A. Cazalilla, R. Citro, T. Giamarchi, E. Orignac, M. Rigol, Rev. Mod. Phys. 83, 1405 (2011).

[30] M.P.A. Fisher, P.B. Weichman, G. Grinstein, D.S. Fisher, Phys. Rev. B 40, 546 (1989). 
[31] M. Greiner, O. Mandel, T. Esslinger, T.W. Hänsch, I. Bloch, Nature 415, 39 (2002).

[32] W.S. Bakr, A. Peng, M.E. Tai, R. Ma, J. Simon, J.I. Gillen, S. Folling, L. Pollet, M. Greiner, Science 329, 547 (2010).

[33] M. Łącki, B. Damski, J. Zakrzewski, Sci. Rep. 6, 38340 (2016).

[34] K. Mayer, A. Rodriguez, A. Buchleitner, Phys. Rev. A 91, 053633 (2015).
[35] E. Bogomolny, Multifractality in simple systems, presentation at the conference Complex patterns in wave functions: drums, graphs, and disorder at the Kavli Royal Society Centre, UK 2012.

[36] S. Ejima, H. Fehske, F. Gebhard, Europhys. Lett. 93, 30002 (2011). 